Revista de la Escuela de Ciencias de la Educación, año 14, nRo. 13, vol. 1, enero a junio de 2018. Páginas 85-103. ISSN 1851-6297 (DESDE DICIEMBRE DE 2006 A DICIEMBRE DE 2017). ISSN 2362-3349 (EN LINEA). FORMACIÓN DOCENTE, NARRATIVAS Y POLITICAS PÚBLICAS CON ROSTRO HUMANO. DEL MAGISTERIO DE EDUCACIÓN BÁSICA (MEB) AL PROYECTO "POLOS DE desarrollo". Luis Gabriel Porta y Jonathan Ezequiel Aguirre.

\title{
FORMACIÓN DOCENTE, NARRATIVAS Y POLÍTICAS PÚBLICAS CON ROSTRO HUMANO. DEL MAGISTERIO DE EDUCACIÓN BÁSICA (MEB) AL PROYECTO “POLOS DE DESARROLLO”
}

\author{
Por Luis Gabriel Porta* \\ CONICET - Universidad Nacional de Mar del Plata, Argentina \\ luisporta510@gmail.com \\ Jonathan Ezequiel Aguirre** \\ CONICET - Universidad Nacional de Mar del Plata, Argentina \\ aguirrejonathanmdp@gmail.com
}

\section{Resumen}

Recibido: 22/08/2017 Aceptado: 9/10/2017

En Argentina, han transcurrido diversos proyectos de formación docente diferenciados y con caracterizaciones propias. En este trabajo recorreremos tres políticas públicas para el sector de la formación docente que a nuestro criterio y al de los especialistas entrevistados, merecen ser recuperadas y puestas en consideración. Entendemos que el Magisterio de Educación Básica (MEB), el Programa de Transformación de la Formación Docente (PTFD) y el Proyecto Polos de Desarrollo, constituyen ejemplos de políticas públicas potentes para la formación de profesores y cuyos rastros hoy se reconocen sedimentados de maneras diversas en las instituciones y en el subsistema formador argentino. Desarrollaremos especialmente el proyecto "Polos de Desarrollo (2000-2001)" a partir de las narrativas de los propios actores y de referentes del campo de la formación docente, con el objetivo de recuperar las políticas públicas desde un enfoque alejado de la concepción clásica, y situado principalmente en los sujetos que las llevan adelante en los propios

* Es Investigador Independiente de CONICET. Departamento de Ciencias de la Educación. Centro de Investigaciones Multidisciplinarias en Educación.

** Es Becario Doctoral de CONICET. Universidad Nacional de Mar del Plata. Departamento de Ciencias de la Educación. Centro de Investigaciones Multidisciplinarias en Educación 
Revista de la Escuela de Ciencias de la Educación, año 14, nRo. 13, vol. 1, enero a junio de 2018. Páginas 85-103. ISSN 1851-6297 (DESDE DICIEMBRE DE 2006 A DICIEMBRE DE 2017). ISSN 2362-3349 (EN LINNEA). FORMACIÓN DOCENTE, NARRATIVAS Y POLITICAS PÚBLICAS CON ROSTRO HUMANO. DEL MAGISTERIO DE EDUCACIÓN BÁSICA (MEB) AL PROYECTO "POLOS DE desarrollo". Luis Gabriel Porta y Jonathan Ezequiel Aguirre.

contextos de concreción, otorgándole a las políticas un rostro cada vez más humano y contextual.

Palabras clave: Educación superior - Políticas públicas - Formación docente.

\begin{abstract}
In Argentina, there have been several different teacher training projects with their own characterizations. In this paper we will look at three public policies for the teacher training sector that in our opinion and that of the specialists interviewed deserve to be recovered and taken into consideration. We understand that the Teachers of Basic Education (MEB), the Teacher Training Transformation Program (PTFD) and the Polos de Desarrollo Project are examples of powerful public policies for the training of teachers and whose traces today are recognized in different ways In the institutions and in the Argentine training subsystem. We will especially develop the project "Poles de Desarrollo (20002001)" based on the testimonies of the actors themselves and of reference in the field of teacher education, with the objective of recovering public policies from an approach far from the classical conception, and located mainly in the subjects that carry them forward in the concrete contexts of concretion, granting to politics an increasingly human and contextual face.
\end{abstract}

Keywords: Higher education - Public politics - Teacher Training.

\title{
Introducción
}

Abordar el análisis de la formación docente en el marco de sus políticas públicas, implica una tarea tan interesante como compleja. Más aún si lo que se recupera son proyectos concretos de políticas que han tenido a la formación de profesores como núcleo central de sus propuestas y han significado verdaderas huellas en el campo formador. Intentamos recuperar en este trabajo tres políticas públicas que, a nuestro criterio, han significado marcados aportes en lo que refiere a la actualización institucional, curricular y normativa de las instituciones formadoras de docentes, como así también en lo que respecta al lugar que le han otorgado a los actores locales en el desarrollo propio de las propuestas. Destacamos aquí que tanto el Magisterio de Educación Básica (MEB), el Programa de Transformación de la Formación Docente (PTFD) como el Proyecto Polos de Desarrollo son parte del entramado de políticas públicas más importante desde finales de la década del '80 en materia de formación docente y cuyos rastros hoy se reconocen de maneras diversas en las instituciones, en los actores y en la memoria del subsistema formador. No es nuestra intención descartar o ignorar las políticas públicas llevadas a cabo a partir del 2003 en materia educativa y de formación docente en nuestro 
Revista de la Escuela de Ciencias de la Educación, año 14, nRo. 13, vol. 1, enero a junio de 2018. Páginas 85-103. ISSN 1851-6297 (DESDE DICIEMBRE DE 2006 A DICIEMBRE DE 2017). ISSN 2362-3349 (EN LíNEA). FORMACIÓN DOCENTE, NARRATIVAS Y POLITICAS PÚBLICAS CON ROSTRO HUMANO. DEL MAGISTERIO DE EDUCACIÓN BÁSICA (MEB) AL PROYECTO "POLOS DE desarrollo". Luis Gabriel Porta y Jonathan Ezequiel Aguirre.

país. Solo hemos hecho una selección, que como toda, siempre es arbitraria. Destacamos las tres primeras políticas de formación desde la restitución democrática en adelante porque son las que surgen de nuestras entrevistas y porque consideramos que sirvieron de base, en gran parte, para los proyectos pedagógicos y políticos que se suscitaron posteriormente en nuestro país.

Como adelantamos en el resumen del artículo, analizaremos con mayor profundidad el programa "Polos de Desarrollo (2000-2001)" que se llevó a cabo durante el gobierno de la Alianza (1999-2001) y que se vio bruscamente interrumpido por la crisis institucional, política y económica vivida en el país en aquellos años. Nos centraremos en dicha política principalmente por tres razones. La primera de ellas porque el proyecto Polos se ha presentado un modo alternativo de transformación de la formación docente y de la manera de concebir e interpretar las políticas públicas para el sector. Nos decía en una entrevista la Asesora Permanente del Programa de Formación Docente (200-2001)

[Polos de Desarrollo] significó mucho. Fue realmente una oportunidad para hacer algo fuerte para el desarrollo de los enfoques institucionales en el país. Fue como una usina de experiencias. Algo que produjo cambio, dotó a los institutos de gente con capacidad de hacer cosas importantes. Pero además fue para mí importante (...) Ir viendo cómo se iban desarrollando algunas de las cuestiones. Se mostró como una herramienta muy importante de intervención en el sentido técnico del término, como un acontecimiento que le permite a la gente volver a mirar su realidad de otro modo y encontrar otras formas para hacer. Pero además fue la puesta en práctica de una interpelación directa a las formas de intervención centralizadas. Polos fue efectivamente un proyecto federal apoyado por una instancia nacional respetuosa de la diversidad regional y cultural de Argentina (Entrevista a Asesora Permanente del Programa Nacional de Formación Docente).

En segundo lugar retomamos la experiencia de Polos de Desarrollo porque luego de casi 17 años y como producto de nuestras investigaciones, reconocemos que en la actualidad se pueden rastrear producciones y experiencias de alto recupero pedagógico, que se desprenden del proyecto iniciado por la Dra. Edith Litwin y su equipo el inicio de los años 2000. Por último nos centramos en dicha experiencia debido a que conforma el objeto de estudio de un proyecto más amplio de investigación en el marco de una Beca de formación doctoral otorgada por CONICET y desarrollada en el Centro de Investigaciones Multidisciplinarias en Educación (CIMED) de la Universidad Nacional de Mar del Plata, cuyo objetivo principal, es interpretar, justamente, las políticas educativas oficiales en Argentina en materia de Formación Docente a partir de un estudio cualitativo del proyecto Polos de Desarrollo presentado anteriormente. 
Revista de la Escuela de Ciencias de la Educación, año 14, nRo. 13, vol. 1, enero a junio de 2018. Páginas 85-103. ISSN 1851-6297 (DESDE DICIEMBRE DE 2006 A DICIEMBRE DE 2017). ISSN 2362-3349 (EN LINNEA). FORMACIÓN DOCENTE, NARRATIVAS Y POLITICAS PÚBLICAS CON ROSTRO HUMANO. DEL MAGISTERIO DE EDUCACIÓN BÁSICA (MEB) AL PROYECTO "POLOS DE desarrollo". luis Gabriel Porta y Jonathan Ezequiel Aguirre.

Nos posicionamos en una perspectiva epistemológica que se aleja del estudio del Estado y de sus políticas públicas educativas de manera lineal, racional y meramente normativa. Más bien recuperamos el sentido y la práctica de las políticas públicas que se resignifican permanentemente por múltiples actores individuales y colectivos que participan de los procesos tanto de revisión de su diseño como de su implementación. De allí la potencia metodológica de reconstruir los proyectos, particularmente Polos de Desarrollo, desde las narrativas de los sujetos participantes. Asimismo, recuperar las políticas públicas desde los sujetos y sus voces, nos habilita a otorgarles a dichas políticas aquellos rostros humanos que las llevan adelante. Esta manera de abordar el objeto de estudio asume una mirada diferente, que repara en los relatos, experiencias y narraciones, en los diversos ámbitos de interlocución, en las contradicciones y zonas grises, "en la multiplicidad de contactos sociales en los que participan quienes son el Estado, quienes son su rostro humano" (Bohoslavsky y Soprano, 2010, p. 39).

\section{Las Políticas Públicas en Formación Docente Creaciones y recreaciones de los sujetos}

En los últimos años, en el debate académico de las Ciencias Sociales en la Argentina se han desplegado nuevas perspectivas teóricas y metodológicas que destacan dimensiones de análisis no atendidas, particularmente, por trabajos previos sobre el estudio del Estado y de las políticas públicas. Si hasta principios de 1980 abundaban los estudios macro-sociológicos que pensaron el objeto de estudio en sus relaciones con la estructura social, nacional e internacional, desde las últimas décadas cobraron vigor nuevas aproximaciones, preocupadas por singularizar proyectos y comprender trayectorias de políticas públicas específicas, considerando al Estado no solo como una totalidad social homogénea, sino atendiendo a la diversidad de actores, instituciones y tensiones que se establecen en la práctica en contextos locales. Estas perspectivas constituyen, a nuestro criterio, un significativo punto de partida para el estudio de políticas públicas educativas centradas en los actores y en las tramas interpersonales que les dan sentido (Bohoslavsky y Soprano, 2010).

De este modo, se puede afirmar que la definición y puesta en marcha de las políticas educativas no se resuelve por una lógica racional burocrática, sino que supone la elección de alternativas (decisiones) que ponen en juego diferentes intereses, contradicciones y conflictos. Gvirtz (2010), pone de manifiesto la centralidad que adquieren los actores que se sitúan entre las instancias de elaboración de las políticas educativas (nivel macro) y las diferentes instituciones educativas (nivel micro). En esta perspectiva teórica resulta importante indagar las decisiones de colaboración que son indispensables para la implementación de la política de avance, investigar acerca de los actores cuya intervención en los diversos puntos de decisión se hace indispensable, 
Revista de la Escuela de Ciencias de la Educación, año 14, nRo. 13, vol. 1, enero a junio de 2018. Páginas 85-103. ISSN 1851-6297 (DESDE DICIEMBRE DE 2006 A DICIEMBRE DE 2017). ISSN 2362-3349 (EN LíNEA). FORMACIÓN DOCENTE, NARRATIVAS Y POLITICAS PÚBliCAS CON ROSTRO HUMANO. DEL MAGISTERIO DE EDUCACIÓN BÁSICA (MEB) AL PROYECTO "POLOS DE Desarrollo". Luis Gabriel Porta y Jonathan Ezequiel Aguirre.

reconocer los puntos de decisión, identificar las aprobaciones que es indispensable que sucedan en su desarrollo, entre otros.

Partimos de la premisa de considerar que la política educativa como campo teórico se define a partir de la perspectiva y el posicionamiento epistemológico de quien la interprete o defina. Con esto decimos, no existen caracterizaciones universales de la política educativa como campo teórico y de su objeto de estudio. Dado que el objeto de estudio de la política educacional se construye de un modo que no es neutral, así el propio desarrollo histórico del campo teórico va definiendo nuevos objetos de estudio o transformando el existente (Di Franco y Siderac, 2016).

El campo de la política educativa (Paviglianiti, 1996), supone concepciones acerca del ser humano, la sociedad, el papel del Estado y de los actores en la orientación y provisión de la educación al conjunto de la población. En este sentido, se pone el acento en la relación entre las estrategias que los individuos utilizan para alcanzar sus intereses, el control institucional y los conflictos que se generan, explicitando ese lado oscuro de lo que sucede en las instituciones educativas en su encuentro con las políticas educativas. Así, Burch (2009) refiriéndose a la investigación en política educacional explica que cada vez se hace más difícil sostener el modelo tradicional del objeto de estudio del campo de la política educacional dado que este campo está cada vez más congestionado: nuevas relaciones y nuevas formas de relación están siendo establecidas en la política y en relación con ella.

Es esencial, en las investigaciones de políticas educativas considerar "las opiniones de los sujetos involucrados en ellas (...) el punto más importante es la naturaleza de la representación y de la conceptualización de las personas en nuestros textos como un todo y en nuestros modelos de sociabilidad" (Ball, 2011, p. 47). Al interpretar las políticas educativas desde la valorización de los sujetos implicados se refleja cómo concebimos el papel de éstos en la sociedad en general y en el contexto de la política en particular, las identidades sociales y colectivas de los sujetos, los formuladores de las políticas, las interacciones que ocurren entre sujetos y las relaciones de poder en los diferentes niveles y escalas (Paviglianiti, 1996).

Este entramado de actores involucrados en el desarrollo del curso de acción que prescribe toda política debe ser precisamente uno de los objetos de toda investigación referida a las políticas públicas ya que, en su implementación, este entramado de actores adquieren protagonismo transformándolas y resignificándolas más allá de la mera implementación de las mismas en el nivel macro. Así, los contenidos de la política dependen de las relaciones y del cruce entre procesos decisorios que involucran a una compleja trama de actores políticos que intervienen en los procesos de implementación de políticas, así como en las sucesivas redefiniciones que se dan en el contento de los entramados de intereses que los propios actores delinean. 
Revista de la Escuela de Ciencias de la Educación, año 14, nRo. 13, vol. 1, enero a junio de 2018. Páginas 85-103. ISSN 1851-6297 (DESDE DICIEMBRE DE 2006 A DICIEMBRE DE 2017). ISSN 2362-3349 (EN LINEA). FoRMACIÓN DOCENTE, NARRATIVAS Y POLITICAS PÚBLICAS CON ROSTRO HUMANO. DEL MAGISTERIO DE EDUCACIÓN BÁSICA (MEB) AL PROYECTO "POLOS DE desarrollo". Luis Gabriel Porta y Jonathan Ezequiel Aguirre.

Las políticas públicas, desde esta posición, son el resultado de tensiones intra-estatales, entre grupos sociales y entre éstos con el Estado. Por ello, es central comprender las apropiaciones y las resistencias que se dan en el terreno de acción, es decir, en el espacio público en donde los sujetos interactúan con diversos niveles estatales independientemente de la normativa vigente. Por esta razón entendemos que los límites de la acción estatal, mediante las políticas públicas, son porosos ya que son el resultado de un proceso permanente de desafíos, restauración y re legitimación protagonizado por personas, grupos, instituciones estatales y no estatales (Bohoslavsky y Soprano, 2010, p. 30). Los contextos locales traducen las políticas de acuerdo a las necesidades propias de la comunidad, la traducción de las políticas en el contexto de la práctica incluye procesos complejos de préstamos, apropiación y adaptación que son hechos por medio de redes de actores/participantes dentro y fuera de la escuela, comprometidos en la colaboración /negociación en diferentes circunstancias (equipos, historias institucionales) y con diferentes formas y volúmenes de recursos (Ball, 2012).

En nuestra investigación, concebimos a las políticas como entramados puestos en acción en condiciones materiales y subjetivas específicas, con variedad de recursos. En este sentido lo material, lo estructural y lo relacional precisan ser incorporados en el análisis de políticas para comprender la política en acción en el nivel institucional local. Retomamos el concepto de entramado porque es precisamente eso lo que se forma cuando la política educativa se estructura en su relación con otros campos de estudio, es allí donde nos encontramos con las epistemologías de la política educativa. De este modo se puede considerar el campo teórico de la política educativa como aquel entramado, entretejido, red en tanto se define como campo de conocimiento que permite nuevas construcciones conceptuales que permiten la interacción múltiple y recíproca entre distintos campos de conocimiento.

Pensamos, entonces, las políticas educativas, específicamente las de formación docente, como un movimiento horizontal, en un continuo de decisiones en donde actores gubernamentales, instituciones, sindicatos, profesores y comunidad local van transformando, de forma progresiva el desarrollo de una acción política. De esto da cuenta la experiencia Polos de Desarrollo, objeto de nuestra investigación. Así, intentamos visibilizar a las políticas con verdadero rostro humano, ya no desde la normativa o el análisis desde los documentos oficiales exclusivamente, sino desde las narrativas y los rostros de las personas que les dan sentido concreto en la región, en la comunidad, en las instituciones y en las aulas.

\section{Definiciones metodológicas}

La presente investigación aspira a interpretar las políticas educativas y las tramas interpersonales que le dan sentido desde una lógica compleja, en su 
Revista de la Escuela de Ciencias de la Educación, año 14, nRo. 13, vol. 1, enero a junio de 2018. Páginas 85-103. ISSN 1851-6297 (DESDE DICIEMBRE DE 2006 A DICIEMBRE DE 2017). ISSN 2362-3349 (EN LINNEA). FORMACIÓN DOCENTE, NARRATIVAS Y POLITICAS PÚBLICAS CON ROSTRO HUMANO. DEL MAGISTERIO DE EDUCACIÓN BÁSICA (MEB) AL PROYECTO "POLOS DE desarrollo". luis Gabriel Porta y Jonathan Ezequiel Aguirre.

multiplicidad, en lo histórico social, en sus supuestos y tradiciones y en las nociones de base que la constituyen (Souto; 2009, 2016). Este análisis plantea la necesidad de una investigación con enfoque cualitativo, interpretativo, narrativo y microsocial. El carácter interpretativo se centra en la comprensión, dado que los hechos nunca hablan por sí solos, sino a través de la experiencia y acción de los sujetos. Esto implica que la realidad se comprende como situada, divergente, dinámica, construida; el énfasis está en la singularidad, la discontinuidad, la ruptura y las diferencias (Gurdián Fernández, 2007).

Esta dinámica de trabajo la ponemos en valor desde el enfoque biográfico-narrativo. Este nos permite retomar las voces y prácticas de los sujetos que intervinieron en los procesos de diseño, puesta en marcha, concreción y evaluación del proyecto Polos de Desarrollo en los diversos contextos locales. La adopción de este enfoque no se limita a una simple metodología para la recogida y análisis de datos, sino que representa un enfoque en sí mismo dentro de la investigación cualitativa (Bolívar, Domingo y Fernández Cruz, 2001). Según Denzin y Lincoln (2008, p.69) "la narrativa, ya sea oral o escrita, es una forma particular del discurso; es la creación de significado en retrospectiva, la configuración o el ordenamiento de la experiencia pasada". La narrativa, entonces, es un modo de comprender las acciones propias y las de los demás, de organizar acontecimientos y objetos en un todo significado y de relacionar y ver las consecuencias de las acciones y los acontecimientos en el tiempo. "Estudiar la política desde un enfoque narrativo aporta nuevas comprensiones, dimensiones y caras invisibles que exigen un nivel de reflexividad del investigador. Esto implica, desprivatizar o desindividualizar lo personal por medio de la narrativa, o personalizar lo político al construir historias" (Bolívar, 2016, p. 345).

En lo que respecta a las políticas educativas en materia de formación docente y a las instituciones formadoras, la narrativa también representa un camino para su interpretación puesto que los sujetos que narran también se desarrollan en instituciones, las cuales por cierto, poseen sus propias narrativas (Domingo Segovia, 2010).

Se utilizó para esta investigación el análisis de documentos, normativa y legislación oficial. Asimismo se realizaron entrevistas en profundidad a gestores, asesores, coordinadores nacionales y jurisdiccionales del proyecto Polos de Desarrollo, en sus diferentes etapas y niveles de responsabilidad, y otras instancias de concreción, prestando atención a sus líneas teóricas, de investigación y de innovación pedagógica en las áreas o campos del conocimiento planteadas. Como parte del trabajo de campo se realizaron instancias presenciales intensivas en diversas Instituciones Sede del proyecto Polos de Desarrollo, allí se recabaron testimonios valiosos para la reconstrucción del objeto de estudio. Por último realizamos entrevistas a especialistas en el campo de políticas públicas en materia de formación docente quienes nos brindaron un panorama global y general de los diferentes programas y proyectos 
Revista de la Escuela de Ciencias de la Educación, año 14, nRo. 13, vol. 1, enero a junio de 2018. Páginas 85-103. ISSN 1851-6297 (DESDE DICIEMBRE DE 2006 A DICIEMBRE DE 2017). ISSN 2362-3349 (EN LINEA). FoRMACIÓN DOCENTE, NARRATIVAS Y POLITICAS PÚBLICAS CON ROSTRO HUMANO. DEL MAGISTERIO DE EDUCACIÓN BÁSICA (MEB) AL PROYECTO "POLOS DE desarrollo". luis Gabriel Porta y Jonathan Ezequiel Aguirre.

seleccionados en el presente trabajo. En suma, hemos realizado a lo largo de todo el proceso de campo de la investigación más de 30 entrevistas. Aquí solo exponemos algunas narraciones que nos parecen enriquecedoras y pertinentes a los objetivos de esta producción.

Si bien, parte de los entrevistados han dado su consentimiento para explicitar sus narrativas, se ha intentado resguardar el anonimato y la confidencialidad de los datos aportados. En tal sentido, el lector podrá identificar tres grupos de entrevistas. En primer lugar las cinco que refieren a los testimonios de los referentes del campo de la formación docente. En segundo lugar recuperamos cuatro relatos del equipo de Polos de Desarrollo (E.P.D) y por último se explicitan algunas de las narrativas de docentes y coordinadores locales del proyecto en los Institutos de Formación con los cuales trabajamos. Cada entrevista se identifica, además, con el número correspondiente.

\section{Políticas intensivas en Formación Docente:}

\section{Las huellas del Magisterio de Educación Básica (MEB) y el Programa de Transformación de la Formación Docente (PTFD)}

Hacia finales de la década de 1980 comenzaron a implementarse políticas públicas nacionales de formación docente que significaron, con diferentes resultados y alcances, propuestas de reforma institucional. En este sentido, cabe ubicar dos transformaciones o reformas sucesivas y que significaron a nuestro criterio potentes políticas públicas en materia de formación docente que dejaron su marca en el derrotero histórico del sector formador. Una de ellas se desarrolló entre los años 1988 y 1990 y se denominó "Magisterio de Educación Básica" (MEB) y la otra se ejecutó entre 1991 y 1995 cuya denominación fue "Programa de Transformación de la Formación Docente" (PTFD).

La Dirección Nacional de Educación Superior, presentó en 1987, un anteproyecto de plan de formación docente para el nivel primario, en donde se empleaba la denominación de "Maestros de Educación Básica" (MEB). En 1988, por resolución ministerial $N^{\circ} 580 / 88$, se divulgó el nuevo plan. El MEB tenía como objetivo, impulsar reformas en las instituciones formadoras de docentes. Se tenía como supuesto de base que no bastaba con realizar modificaciones curriculares sino que las instituciones debían transformarse para que sus egresados pudieran generar una escuela diferente (Diker y Terigi, 1997). Desde esta perspectiva, se aspiró a que los ISFD "debían enseñar no solo conocimientos sino que debían trabajar con los futuros docentes para reflexionar sobre sus concepciones acerca de qué constituía un buen docente, y de esta manera generar actitudes diferentes y competencias específicas del acto de enseñar" (Ruiz, 2011, p.30). En una entrevista que concediera Ovide Menin a las coordinadoras del Programa Nacional de Formación Docente en el año 2000 explicaba lo siguiente, 
Revista de la Escuela de Ciencias de la Educación, año 14, nRo. 13, vol. 1, enero a junio de 2018. Páginas 85-103. ISSN 1851-6297 (DESDE DICIEMBRE DE 2006 A DICIEMBRE DE 2017). ISSN 2362-3349 (EN LINEAA). FORMACIÓN DOCENTE, NARRATIVAS Y POLITICAS PÚBLICAS CON ROSTRO HUMANO. DEL MAGISTERIO DE EDUCACIÓN BÁSICA (MEB) AL PROYECTO "POLOS DE desarrollo". Luis Gabriel Porta y Jonathan Ezequiel Aguirre.

EI MEB fue un proyecto de formación de maestros para la Educación Básica que consistió en centrar en la institución formadora por excelencia, la Escuela Normal, un programa de acción fuertemente autónomo. Se inició en 1988 y la última cohorte de graduados obtuvo su título en 1992. El recorrido curricular duraba cuatro años consecutivos, abarcaba los dos últimos años del nivel medio y dos del nivel terciario y estuvo estructurado en cinco áreas de conocimiento: Ciencias del Lenguaje, Ciencias Sociales, Ciencias Naturales, Matemáticas y Ciencias de la Educación. El cuarto año se destinó a las residencias docentes intensivas, tanto en Educación formal, como en Educación no Formal y de Iniciación en la Práctica Investigativa, preferiblemente de campo. (Entrevista al Dr. Ovide Menin, diciembre de 2000).

El programa fue iniciado durante la gestión en Educación Superior del Dr. Menin en 1988. Por resolución ministerial N¹679, se resolvió transferir el ciclo de educación secundaria de 154 escuelas normales, que hasta entonces dependían de la Dirección Nacional de Educación Media, a la Dirección Nacional de Educación Superior.

El programa trabajó en el esfuerzo por resolver los problemas de articulación existentes entre el nivel medio y superior. Su filosofía formadora daba inicio en espacios curriculares que correspondían a los últimos años del entonces nivel medio y que acreditaban bajo la expedición del diploma de bachiller con orientación pedagógica. De esta manera, la formación docente en el MEB, empezaba en cuarto y quinto año y luego se orientaba hacia el área pedagógica.

Recuerdan dos de las referentes en el campo de la Formación Docente sobre el proyecto en cuestión,

Apenas él asume como Director Nacional del Nivel Superior, lo hace para la formación de los docentes de Nivel Inicial y Primaria. Porque ya era evidente que las carreras de formación debían tener 4 años. Todavía no estaban dadas las posibilidades institucionales ni económicas. Entonces él crea ese proyecto de transición alternativo que fue interesantísimo. Los chicos que hacían la docencia empezaban a formarse en 4to año de secundario y después se formaban dos años más. Los contenidos estaban organizados en áreas, a partir de una concepción curricular muy novedosa. Los graduados de ese plan, que duró poco tiempo, recuperan muchísimo esa experiencia. (Referente del campo de Formación Docente №1).

La experiencia del MEB recupera la formación docente en el último ciclo de la escuela media y la hace de 4 años. Me parece que el MEB fue un muy buen proyecto, sobre todo por el momento en el que se hizo. El momento permitía producir alguna innovación en la formación y era una forma también de permitir innovaciones curriculares, en cuanto al tipo de contenido, la articulación del contenido las teorías didácticas y pedagógicas que sustentaban el proyecto. (Referente del campo de Formación Docente №2). 
Revista de la Escuela de Ciencias de la Educación, año 14, nRo. 13, vol. 1, enero a junio de 2018. Páginas 85-103. ISSN 1851-6297 (DESDE DICIEMBRE DE 2006 A DICIEMBRE DE 2017). ISSN 2362-3349 (EN LINEA). FoRMACIÓN DOCENTE, NARRATIVAS Y POLITICAS PÚBLICAS CON ROSTRO HUMANO. DEL MAGISTERIO DE EDUCACIÓN BÁSICA (MEB) AL PROYECTO "POLOS DE desarrollo". Luis Gabriel Porta y Jonathan Ezequiel Aguirre.

El programa antes descripto, produjo dos promociones de egresados y se canceló en 1990 por disposición del gobierno nacional (Menin, 1998). A pesar de su corta duración, se podría sostener, que el MEB constituyó un primer intento de política nacional innovadora para la formación docente, luego de casi dos décadas sin políticas sectoriales específicas que cubrieran aspectos institucionales y curriculares del sistema formador. Al respecto uno de nuestros entrevistados recupera al MEB desde su carácter de innovador para la época,

[fue innovador] porque en ningún proyecto anterior apareció marcada con tanta fuerza la libertad para pensar sin cortapisas formales. Considero que fue innovador porque brindó a los actores la oportunidad de intervenir de manera franca en la crítica, la renovación y el balance institucional. Fue innovador porque, además de recoger recomendaciones de los especialistas, dejó amplios espacios libres para que el grupo humano convocado a hacer la experiencia, piense y re piense qué se hacía en cada institución. (Referente del campo de Formación Docente $N^{\circ} 3$ ).

En este relato se ve claramente la huella de una política pública intensiva y situada en los actores, y donde la voz de los sujetos es retomada para mejorar, evaluar y repensar los términos de la política a niveles normativos. Su innovación radicó en parte, por la libertad de discusión en los niveles locales de aplicación. Una política con rostro humano.

El segundo proyecto que abordaremos aconteció entre 1991 y 1995 en un clima previo a las políticas de transferencia educativa a las provincias que se implementarán como parte de las reformas propiciadas por el Estado con alto impacto en el sector formador. El Programa de Transformación de la Formación Docente (PTFD) partió de cierto cuadro de situación que se fue precisando a medida que el trabajo con las instituciones formadoras permitió el debate y la incorporación de aspectos nuevos (Diker y Terigi, 1995). El diagnóstico con el que se contaba enfatizaba en tres puntos cruciales sobre la situación de la formación docente. El primero de ellos era una severa distancia que tenía la formación docente con las prácticas para las que se formaba. El segundo punto fue la tradicional fragmentación y segmentación de la oferta docente. Existían circuitos diferenciados curricularmente para la formación de grado de docentes sin áreas comunes de formación. Y el tercer punto estaba referido a la organización institucional, ya que los circuitos de formación docente tendían a centrarse exclusivamente en la formación de grado. El perfeccionamiento y la actualización, no estaban sistemáticamente previstos ni provistos por el sistema (Diker y Terigi, 1995). En este marco de situación el Ministerio de Educación Nacional, inició en 1991 su principal política de reforma para las instituciones superiores (R.M N²547/91).

Dicho programa no se planteó, desde sus objetivos, como un nuevo plan de estudios de formación docente. Tampoco como un plan de capacitación de 
Revista de la Escuela de Ciencias de la Educación, año 14, nRo. 13, vol. 1, enero a junio de 2018. Páginas 85-103. ISSN 1851-6297 (DESDE DICIEMBRE DE 2006 A DICIEMBRE DE 2017). ISSN 2362-3349 (EN LINNEA). FORMACIÓN DOCENTE, NARRATIVAS Y POLITICAS PÚBLICAS CON ROSTRO HUMANO. DEL MAGISTERIO DE EDUCACIÓN BÁSICA (MEB) AL PROYECTO "POLOS DE desarrollo". Luis Gabriel Porta y Jonathan Ezequiel Aguirre.

docentes en ejercicio. En un sentido muy específico, desde sus comienzos, el PTFD, se planteó como un programa que procuraba una transformación integral de la formación docente: pretendió avanzar progresivamente sobre las diversas cuestiones que caracterizaban la situación descripta. EI PTFD intentó plasmar una estrategia que abarcaba al conjunto de la formación docente y sus problemas. El programa ejecutó acciones, luego de la ley de transferencias de los servicios públicos (Ley 24049/92) en 36 institutos superiores ex nacionales y en 25 institutos provinciales. Estas acciones estuvieron dirigidas durante los primeros dos años a generar instancias de trabajo con instituciones en función de tres etapas. Producir un diagnóstico conjunto de la situación de la formación docente, en segundo lugar consensuar una estructura curricular capaz de dar respuestas a esos problemas detectados y por ultimo acordar las acciones que habrían de implementarse para iniciar la transformación (Diker y Terigi, 1995). Refiriéndose al PTFD, uno de nuestros entrevistados lo recuerda destacando la transformación que proponía,

Era un programa nacional que trata de pensar integralmente, no provincialmente, a los Institutos y plantea una transformación muy grande porque esa transformación pedagógica era sostenida por una transformación institucional. Reordenar el trabajo dentro de los institutos organizados por departamentos, ahí tomando las estructuras de las universidades. Departamentos de extensión, investigación y formación... departamentaliza el trabajo. Y empiezan a trabajar en esa línea. (Referente del campo de la Formación Docente $\mathrm{N}^{\circ} 4$ ).

Repiensan algunas cuestiones de formas muy interesantes, refocalizando todo el trabajo en relación a la enseñanza. Profesionalizando a los institutos [...] había mucha bibliografía, muchas reuniones con los institutos, había mucha producción de materiales súper interesantes [...] (Referente del campo de la Formación Docente $N^{0} 1$ ).

Como argumentan las narrativas de los entrevistados, el Programa redefinía las funciones de los ISFD con la inclusión de la capacitación y la investigación. Sus objetivos y lineamientos de política se plasmaron en el documento "Propuesta Curricular Institucional para la Formación Docente Continua", aprobado como base para la discusión nacional en el CFCyE (Consejo Federal de Cultura y Educación) de 1992.

Uno de los aspectos de la transformación institucional que llevó adelante el PTFD fue la inclusión de nuevas actividades para los institutos superiores. El programa proponía la inclusión de departamentos de extensión, investigación y docencia al estilo de las estructuras de las universidades. Cabe aclarar que estos departamentos no fueron pensados como espacios institucionales aislados unos con otros, creados al solo fin de organizar mejor las nuevas funciones que asumía el instituto formador. Por el contrario se trataba de generar 
Revista de la Escuela de Ciencias de la Educación, año 14, nRo. 13, vol. 1, enero a junio de 2018. Páginas 85-103. ISSN 1851-6297 (DESDE DICIEMBRE DE 2006 A DICIEMBRE DE 2017). ISSN 2362-3349 (EN LINNEA). FORMACIÓN DOCENTE, NARRATIVAS Y POLITICAS PÚBLICAS CON ROSTRO HUMANO. DEL MAGISTERIO DE EDUCACIÓN BÁSICA (MEB) AL PROYECTO "POLOS DE desarrollo". luis Gabriel Porta y Jonathan Ezequiel Aguirre.

las condiciones que permitieran tanto desde el diseño curricular como desde la organización institucional, abordar la formación continua de maestros en sus diversas instancias de manera de potenciar la especialización de los ISFD en los problemas de la práctica y la formación de maestros.

EI PTFD fue desvirtuándose por razones económicas y por la propia decisión del gobierno nacional de transferir los servicios educativos a las provincias. La descentralización educativa significó el primer cimbronazo en lo que respecta a políticas públicas del sector formador (Ruiz, 2011). La completa transferencia de los servicios educativos nacionales resultó ser una de las decisiones más apresuradas del año 1992 y sorprendió a los responsables del Programa. El resultado fue el desmantelamiento del PTFD al cabo de pocos años (Ruiz, 2011).

De todos modos, hay un amplio consenso en afirmar que dicho programa marcó de sobremanera las instituciones formadoras y que si bien formalmente no continuó como política pública, muchas de las reformas consecuentes han sido posibles por la base institucional que dejó el PTFD. Ambas políticas de formación docente significaron los preliminares de un cambio en los modos de regulación en relación a la formación existente. Al respecto nos dice una de las coordinadoras del proyecto Polos,

EI PTFD en particular fue muy impresionante, tanto que después, muchas de las cuestiones como la recuperación de ciertas disciplinas, de los campos, el tipo de relaciones entre teoría y práctica, el papel de los actores, los vínculos con las instituciones donde se generaban los procesos de la práctica se recuperaron. (Referente del campo de la Formación Docente $N^{\circ} 5$ ). La gente de los Institutos, destacaban mucho lo que había sido el MEB que había iniciado Ovide [Menin], y el PTFD que había trabajado Flavia Terigi. Esos son los proyectos que yo recuerdo que la gente destacaba y reconocía como de impacto curricular, que fueron antecedentes. Y después con los polos también algunos eran reticentes en el inicio, pero Edith [Litwin] tenía la certeza de que eso potenciaría y fortalecería a los institutos... (Referente del campo de la Formación Docente $\mathrm{N}^{\circ} 2$ ).

\section{El Proyecto Polos de Desarrollo en el marco del Programa Nacional de Formación Docente (2000-2001)}

El proyecto Polos de Desarrollo fue parte integral del "Programa Nacional de Formación Docente" del Ministerio de Cultura y Educación de la Nación Argentina, coordinado por la Dra. Edith Litwin y su grupo de trabajo a principios del año 2000. De la normativa analizada surge como principal objetivo el fortalecimiento y el enriquecimiento de la formación docente y de las instituciones formadoras. Cabe aclarar que dichas instituciones se encontraban "estalladas" (Fernández, 1999, p. 25) producto de las políticas de acreditación, 
Revista de la Escuela de Ciencias de la Educación, año 14, nRo. 13, vol. 1, enero a junio de 2018. Páginas 85-103. ISSN 1851-6297 (DESDE DICIEMBRE DE 2006 A DICIEMBRE DE 2017). ISSN 2362-3349 (EN LINEAA). FoRMACIÓN DOCENTE, NARRATIVAS Y POLITICAS PÚBliCAS CON ROSTRO HUMANO. DEL MAGISTERIO DE EDUCACIÓN BÁSICA (MEB) AL PROYECTO "POLOS DE desarrollo". Luis Gabriel Porta y Jonathan Ezequiel Aguirre.

privatización y ajuste vividas en el período prexistente.

A partir del estímulo de propuestas educativas innovadoras y con alto recupero pedagógico, el proyecto aspiró a potenciar la formación de los futuros maestros proponiendo redes de trabajo colaborativo entre las instituciones educativas regionales a través de líneas de especialización que los propios actores locales escogían. El proyecto Polos, desde su marco conceptual, comprendía que era desde la colaboración y la ayuda mutua desde donde se mejoraría la calidad de la educación (Fullan, 2016).

Teniendo en cuenta el contexto noventista de ajuste y corrimiento del estado, lo primero que se hizo, desde la gestión de la Dra. Litwin como Directora Nacional de Educación Superior, fue interrumpir el proceso de acreditación y analizar el impacto que venía teniendo esta política en el seno de los ISFD.

Luego de realizar el diagnóstico y suspender el proceso de acreditación, se generó una política con el objetivo de fortalecer nuevamente a los institutos formadores y otorgarle mayor profesionalización y apoyo estatal. Una de las líneas de la política de formación docente fue encargada al proyecto Polos de Desarrollo. Así, la estrategia que se implementó en el marco del Programa Nacional de Formación Docente fue la de conformar redes solidarias de trabajo y poder lograr la articulación de todas las propuestas pedagógicas que enseñen a romper el aislamiento como manera constitutiva del trabajo docente. Señala una de las coordinadoras del proyecto,

Era una lógica de trabajo en red. Un Instituto no puede ser una isla, es un instituto en el marco de una articulación interesante con otras organizaciones. Pensemos la formación a partir de una línea de especialización y una articulación en función de esa especialización. Entonces, lo que dijimos fue: creemos una línea de desarrollo en donde un instituto sea capaz de trabajar con otros, que esté abierto a trabajar con otros. Se especialice en una línea y genere materiales que son en beneficio, fundamentalmente, de las escuelas y de las organizaciones que se articula. (Coordinadora del Proyecto Polos de Desarrollo- E.P.D N¹).

Para la implementación del proyecto se seleccionaron 84 institutos de formación docente que trabajaron en red con instituciones asociadas: una universidad nacional mediante alguna unidad académica (una cátedra, un departamento, un instituto o una facultad); más de una escuela, todas ellas próximas geográficamente al polo, pero de características sustantivamente diversas como campo de acción educativa, y otros institutos en una acción concertada con las jurisdicciones. La selección de las instituciones que se incorporaron al proyecto requirió acciones de diferente tipo-efectuadas por dichas jurisdicciones- que permitieron identificar a las instituciones que desarrollan auténticas propuestas de envergadura académica y pedagógica. Se 
Revista de la Escuela de Ciencias de la Educación, año 14, nRo. 13, vol. 1, enero a junio de 2018. Páginas 85-103. ISSN 1851-6297 (DESDE DICIEMBRE DE 2006 A DICIEMBRE DE 2017). ISSN 2362-3349 (EN LÍNEA). FORMACIÓN DOCENTE, NARRATIVAS Y POLITICAS PÚBLICAS CON ROSTRO HUMANO. DEL MAGISTERIO DE EDUCACIÓN BÁSICA (MEB) AL PROYECTO "POLOS DE desarrollo". Luis Gabriel Porta y Jonathan Ezequiel Aguirre.

trató de dar impulso a acciones dirigidas a potenciar los desarrollos contenidos y favorecer la cooperación entre instituciones, entendiendo que los nuevos vínculos que se establecerán permitirán la ayuda mutua y el mejoramiento de las prácticas educativas.

Así, cada institución se convirtió en la sede del Proyecto Polo de Desarrollo que aspiró a consolidar un perfil propio, situado y contextualizado, orientado hacia la búsqueda de buenas propuestas pedagógicas para la formación de docentes, y que brindó diversos hallazgos recientes de la investigación en el área de la pedagogía, la didáctica o las disciplinas enseñadas. El Instituto Sede- se convirtió en el nodo de una red de instituciones; supuso la interconexión electrónica entre las instituciones asociadas y constituyó una propuesta experimental que se analizó por especialistas en el campo de la educación.

Los proyectos se agruparon en torno a siete líneas de especialización: Práctica docente (39\%), Didácticas Específicas y Regímenes Especiales (18\%), Diversidad Cultural (14\%), Curriculum (11\%), Tecnología Educativa (9\%), Aprendizaje y fracaso escolar (6\%) y Aspectos Institucionales (3\%) (Anexo Informe de Gestión. Diciembre 2001. MCE de la Nación). La definición de la línea en la que el polo se desarrolló, ya sea por tradición o por elección de sus actores, fue de carácter fundante. Asimismo cada Instituto Polo escogía un referente académico, reconocido por sus aportes en el campo de la formación docente que sería de acompañante y guía en la formulación de las propuestas pedagógicas. Estos referentes eran elegidos por las instituciones de acuerdo a las líneas de especialización seleccionadas.

En suma, el proyecto procuró la participación genuina de los actores involucrados por medio de redes solidarias de intercambio entre las instituciones y los propios sujetos de la comunidad en general, entendiendo a la estructura federal del sistema educativo argentino y a la importancia clave de la Formación Docente para el mejoramiento del sistema en general. De las narrativas de los propios gestores del proyecto emana el sentido ideológico, político y epistemológico con el que se construyó el proyecto.

Una de las cosas más importante del proyecto Polos no era tanto su nombre, sino lo que está debajo del nombre, por eso destaco sus raíces. Esa visión de una institución en red. El proyecto Polos de Desarrollo no era una política que se bajaba desde ningún programa ministerial. Todo un año de apoyatura y de trabajo a la concepción de esa idea que permitía luego una especialidad o una línea de trabajo en el proyecto Polos. Todo un año en donde la preparación tenía que ver con quiénes somos, qué necesitamos, qué nos interesa saber, qué tipos de políticas queremos desarrollar, qué tipo de proyecto educativo y con quiénes queremos llevarlo adelante. (Entrevista a Mariana Maggio. Coordinadora del Proyecto Polos de DesarrolloE.P.D N¹). 
Revista de la Escuela de Ciencias de la Educación, año 14, nRo. 13, vol. 1, enero a junio de 2018. Páginas 85-103. ISSN 1851-6297 (DESDE DICIEMBRE DE 2006 A DICIEMBRE DE 2017). ISSN 2362-3349 (EN LINNEA). FORMACIÓN DOCENTE, NARRATIVAS Y POLITICAS PÚBLICAS CON ROSTRO HUMANO. DEL MAGISTERIO DE EDUCACIÓN BÁSICA (MEB) AL PROYECTO "POLOS DE desarrollo". luis Gabriel Porta y Jonathan Ezequiel Aguirre.

En ese sentido también fue una política de vanguardia, porque buscaba encontrar la voz de los institutos de formación docente y el sentido que podía llegar a tener comunitario y contextual, el desarrollo, la investigación y la experimentación en torno a un proyecto específico. (E.P.D Nº 3 ).

La potencia de Polos fue, que si bien era una propuesta nacional, tenía una lógica política, pedagógica e ideológica en su diseño, lo que sostenía la filosofía del proyecto, era que aunque era una política nacional, el valor y para que funcionara tenía que ver con que los actores se lo apropiaran y quisieran hacer eso. Había una apuesta muy fuerte de Edith [Litwin] y en el equipo de que era posible, de que los actores se podían apropiar, y así lo hicieron. (E.P.D No 2).

Como sostienen las narrativas de los actores, la propuesta del Proyecto Polos de Desarrollo, se enmarcó en un conjunto de criterios y decisiones generales de política educativa comprometida con una gestión democrática y pluralista de la educación superior. Desde este posicionamiento teórico, epistemológico y político se intentó generar una participación democrática a través del trabajo conjunto y colaborativo entre directivos, docentes y futuros docentes entre instituciones del mismo territorio geográfico como lo son las universidades nacionales, las escuelas primarias y secundarias, ONGs, sindicatos, etc.

Según los testimonios relevados y la normativa recopilada, este proyecto se pensó alejado de una lógica de control de procesos y tiempos del trabajo cotidiano al interior de las Instituciones, especialmente a las que remiten a la burocratización de las tareas, a la implementación de los cambios según lógicas verticales de arriba hacia abajo de afuera hacia dentro de las instituciones. Esto se asemejaría a las propuestas macro-políticas clásicas. Más bien sostuvo una política dirigida a potenciar el trabajo colaborativo, situado, contextualizado en el territorio y contando con la participación de los actores involucrados.

Cuando entrevistamos a los miembros de los equipos Polos de Desarrollo de los Institutos de Formación Docente que se constituyeron en sede del proyecto y que conforman el objeto de estudio de nuestra tesis nos decían al respecto,

El proyecto Polos de Desarrollo lo que nos dio fue la posibilidad de unificar todo y darle forma como proyecto fuerte de innovación y de mejoramiento de la didáctica de las Ciencias Naturales. (Docente de ISFD- Miembro del Equipo Polos de Desarrollo).

El mismo Proyecto Polos es algo que volvería hacer, porque para nosotros fue una experiencia importantísima. Nos permitió innovar en nuestra propia práctica como formadores, pero a la vez lo hicimos junto a los estudiantes y junto a las instituciones que visitamos y que nos visitan. Cuando uno habla de trabajo colaborativo, creo que es eso. Trabajar pensando que con el 
Revista de la Escuela de Ciencias de la Educación, año 14, nRo. 13, vol. 1, enero a junio de 2018. Páginas 85-103. ISSN 1851-6297 (DESDE DICIEMBRE DE 2006 A DICIEMBRE DE 2017). ISSN 2362-3349 (EN LÍNEA). FoRMACIÓN DOCENTE, NARRATIVAS Y POLITICAS PÚBLICAS CON ROSTRO HUMANO. DEL MAGISTERIO DE EDUCACIÓN BÁSICA (MEB) AL PROYECTO "POLOS DE desarrollo". Luis Gabriel Porta y Jonathan Ezequiel Aguirre.

otro uno puede mejorar y puede potenciar el trabajo. De modo que en mi vida significó una experiencia lindísima y coordinarla me dio mucho placer. (Docente del ISFD - Coordinadora del Equipo Polos de Desarrollo).

Puedo asegurar que [El proyecto Polos de Desarrollo] se trató efectivamente de una propuesta participativa, y preocupada por el trabajo colaborativo y contextual. En relación con esto último, en la extensa geografía de nuestra provincia [Santiago del Estero], la reunión de los institutos por regiones fue un gran acierto. (Docente de ISFD -miembro del equipo Polos de Desarrollo).

Básicamente el fuerte trabajo colaborativo entre instituciones y el trabajo en equipo por parte de los actores que formaron parte. Pero también la socialización de las diferentes propuestas. Por ahí a veces uno por la dinámica o la característica del instituto en donde trabaja, o en el proyecto que se desarrolla queda ajena a otras experiencias. (Docente del ISFD- Ex alumna Miembro del Equipo Polos de Desarrollo).

El Polo de Desarrollo era pensar ese espacio de una institución educativa que se transforma en algo que puede generar un proyecto que le da una vida mucho más rica, más calidad a la formación y a la vez convoca, comparte con otras instituciones, como universidades, escuelas y ayuda a desarrollar también a otros, hacia dentro y hacia fuera. (Docente de ISFDCoordinadora del Equipo Polos de Desarrollo).

Polos de Desarrollo se mostró como una herramienta que le permitió a la gente volver a mirar su realidad de otro modo y encontrar otras formas para hacer. Pero además fue la puesta en práctica de una interpelación directa a las formas de intervención centralizadas. Polos fue efectivamente un proyecto federal apoyado por una instancia nacional respetuosa de la diversidad regional y cultural de Argentina. (Docente de ISFD- Miembro del Equipo Polos de Desarrollo).

Las narrativas presentan a la política pública de Formación Docente denominada "Polos de Desarrollo" como una propuesta con alto recupero pedagógico aprovechada y resignificada por los propios actores de las instituciones. Es por ello que a partir de los núcleos de sentido que se desprenden de dichos relatos se puede apreciar la relevancia del trabajo colaborativo entre instituciones, el respeto por las diversas realidades regionales y culturales de nuestro país y la significatividad de revisar y reflexionar sobre las propias prácticas cotidianas y los desafíos concretos, para que de ellos surjan proyectos que tiendan a fortalecer y al mismo tiempo enriquecer la formación de futuros profesores. De alguna forma estas narrativas invitan a poner en debate las características que asumen las políticas públicas que están destinadas a la educación y particularmente a la formación docente. Es altamente significativo cuando las políticas emanan desde los contextos y desde las inquietudes par- 
Revista de la Escuela de Ciencias de la Educación, año 14, nRo. 13, vol. 1, enero a junio de 2018. Páginas 85-103. ISSN 1851-6297 (DESDE DICIEMBRE DE 2006 A DICIEMBRE DE 2017). ISSN 2362-3349 (EN LíNEA). FORMACIÓN DOCENTE, NARRATIVAS Y POLITICAS PÚBLICAS CON ROSTRO HUMANO. DEL MAGISTERIO DE EDUCACIÓN BÁSICA (MEB) AL PROYECTO "POLOS DE Desarrollo". Luis Gabriel Porta y Jonathan Ezequiel Aguirre.

ticulares de docentes y de los estudiantes, porque son ellos, en definitiva, los que están inmersos en el cotidiano de las instituciones educativas creándolas y recreándolas constantemente.

\section{Reflexiones finales}

Al comienzo del artículo advertimos, nuestra intención de recuperar e interpretar tres políticas públicas en materia de formación docente, que a nuestro criterio, se convirtieron en propuestas altamente significativas para el sector formador. Fueron los propios sujetos quienes las distinguieron en sus narrativas como experiencias potentes en términos de formación situada en los contextos. No es intención del presente trabajo descartar o ignorar lo acontecido en materia de políticas de formación de maestros inicial y continua desde el 2003 en adelante, en donde, tanto el programa de Renovación Pedagógica (2004-2005) como la creación del INFOD en el año 2007 sirvieron para darle impulso, fortalecer y potenciar el campo. Pero consideramos que tanto el MEB, como el PTFD y Polos de Desarrollo se transformaron en políticas intensivas que se situaron en los contextos locales e intentaron fortalecer las prácticas y dinámicas institucionales desde una forma de implementar la política que a priori se manifestó diferente.

Al mismo tiempo, quisimos desarrollar con mayor profundidad la experiencia de los Polos de Desarrollo para poder así, recuperarla y valorar su huella en el camino del sector formador. Durante estos 17 años poco se ha mencionado sobre esta propuesta que anticipó, no sólo las escuelas asociadas sino que, le dio una impronta democrática, colaborativa y en red a la política de formación docente. El abordaje narrativo, microsocial e interpretativo nos habilitó a repensar nuestros presupuestos y sostener que a pesar de la interrupción de la gestión a nivel nacional en el año 2001, y que fue 'clausurado' por el pragmatismo político y sus objetivos coyunturales, el proyecto Polos de Desarrollo continuó en las instituciones por su fuerte apuesta por los procesos locales de toma de decisión, por estimular la participación de diversos actores educativos de la comunidad y por potenciar la generación de ideas que sean significativas de acuerdo a las necesidades y características de los propios institutos.

Recuperar estas experiencias desde las voces de sus coordinadores, directores y sujetos locales hace comprender aún más a las políticas no como un conjunto de documentos y normativas oficiales, sino como un proceso complejo, dinámico y dialéctico, en donde los actores y los contextos son claves para su interpretación, creando y recreando las políticas desde un plano subjetivo, contextual y experiencial. Esto significa otorgarle rostro humano a las políticas públicas y escapar de análisis tradicionales normativos. Las políticas son llevadas adelante por sujetos que sienten, piensan, viven y narran sus propias experiencias. De allí el potencial metodológico de la narrativa en la reconstrucción de políticas públicas educativas. 
Revista de la Escuela de Ciencias de la Educación, año 14, nRo. 13, vol. 1, enero a junio de 2018. Páginas 85-103. ISSN 1851-6297 (DESDE DICIEMBRE DE 2006 A DICIEMBRE DE 2017). ISSN 2362-3349 (EN LÍNEA). FoRMACIÓN DOCENTE, NARRATIVAS Y POLITICAS PÚBLICAS CON ROSTRO HUMANO. DEL MAGISTERIO DE EDUCACIÓN BÁSICA (MEB) AL PROYECTO "POLOS DE desarrollo". luis Gabriel Porta y Jonathan Ezequiel Aguirre.

\section{Notas bibliográficas}

(1) La caracterización de las Políticas Públicas con Rostro Humano se desprende de la obra de Bohoslavsky E. y Soprano G. (2010) "Un Estado con Rostro Humano" Buenos Aires: Prometeo Libros. Allí los autores analizan los funcionarios y las instituciones estatales en Argentina desde 1880 a la actualidad desde análisis histórico e historiográfico con una perspectiva micro-social, local y desde los propios sujetos.

\section{Referencias bibliográficas}

- Ball, S. (2011). Política social y educativa, empresa social, hibridación y nuevas comunidades discursivas. Propuesta Educativa, vol. 2, (36), pp. 25 a 34.

- Ball, S. (2012). How schools do policy: policy enactments in secondary schools. London: Routledge.

- Bohoslavsky, E. y Soprano. G. (2010). Un Estado con rostro humano. Funcionarios e instituciones estatales en Argentina (desde 1880 a la actualidad). Buenos Aires: Prometeo Libros.

- Bolívar A.; Domingo, J. y Fernández Cruz, M. (2001). La investigación biográficonarrativa en educación. Enfoque y metodología. Madrid: La Muralla.

- Bolivar, A. (2016). Conjugar lo personal y lo político en la investigación (auto)biográfica: Nuevas dimensiones en la política educacional. En: Revista Internacional de Educación Superior. Campinas. Vol 2 №2. pp. 341-365.

- Burch, P. (2009). Hidden Markets: the new education privatization. Abingdon: Routledge.

- Davini, M. C. (2015). Estudio de Calidad y Cantidad de oferta de la formación docente, investigación y capacitación en la Argentina. Ciudad Autónoma de Buenos Aires: Ministerio de Educación de la Nación.

- Denzin, N. K. (2008). La política y la ética de la representación pedagógica: Hacia una pedagogía de la esperanza. En Mc Laren, P. y J. Kincheloe (ed.) Pedagogía Crítica. De qué hablamos, dónde estamos. Barcelona: Graó.

- Di Franco, G. y Siderac, S. (2016). La formación docente en las políticas públicas: el campo de las prácticas como posibilidad. En Revista Práxis y Saber. Vol. 7. №15. pp. 17-40.

- $\quad$ Diker, G. y Terigi, F. (1995). EI PTFD: Un balance todavía provisorio pero ya necesario. En: Revista del Instituto de Investigaciones en Ciencias de la Educación. UBA. Año IV, No7. pp. 78-91.

- Diker, G. y Terigi, F. (1997). La formación de maestros y profesores. Hojas de ruta. Buenos Aires: Paidós.

- Domingo Segovia, J. (2010). Aprendiendo de la experiencia: memoria institucional y relatos de vida de la escuela. En: Revista de Educación. Año 1 №1. pp. 123-140.

- Fernández, A. (1999). Instituciones Estalladas. Buenos Aires: Eudeba.

- Fullan, M. (2016). Indelible leadership: Always leave them learning. Thousand Oaks, CA: Corwin Press

- Gvirtz, S. (2010). Los horizontes de la investigación en políticas educativas: los niveles intermedios y la ampliación del campo. En: Wainerman, C. Di Virgilio MM. El quehacer de la investigación en educación. Universidad de San Andrés. Buenos Aires: Manantial

- Gurdián Fernández, A. (2007). El paradigma cualitativo en la investigación socio- 
Revista de la Escuela de Ciencias de la Educación, año 14, nRo. 13, vol. 1, enero a junio de 2018. Páginas 85-103. ISSN 1851-6297 (DESDE DICIEMBRE DE 2006 A DICIEMBRE DE 2017). ISSN 2362-3349 (EN LINEA). FORMACIÓN DOCENTE, NARRATIVAS Y POLITICAS PÚBLICAS CON ROSTRO HUMANO. DEL MAGISTERIO DE EDUCACIÓN BÁSICA (MEB) AL PROYECTO "POLOS DE desarrollo". luis Gabriel Porta y Jonathan Ezequiel Aguirre.

educativa. Costa Rica: Investigación y Desarrollo Educativo Regional (IDER).

- Jaime, M.; Dufour, G. y Alessandro, M. (2013). Introducción al Análisis de Políticas Públicas. Buenos Aires: Universidad Arturo Jauretche

- Litwin, E. (2001). Argentina: Lineamientos nacionales para la formación del personal docente. En: Revista Perspectivas. Vol 31. №1. pp. 112-119

- Menin, O. (1998). Una experiencia alternativa de formación docente: el MEB. En: Birgin, A. (comp). La formación docente. Cultura, escuela y política. Debate y experiencias. Buenos Aires: Troquel.

- Paviglianiti, N. (1996). Aproximaciones al desarrollo histórico de la Política Educacional. Buenos Aires: CEFyL, UBA.

- Porta, L. (2010). Relatos de buenos profesores universitarios. Aportes de la investigación narrativa en la educación superior. En: Pisano María et al. El estudio de la investigación educativa, Perspectivas latinoamericanas. Córdoba: Ed. de la Universidad Católica de Córdoba.

- Ruiz, G. (2011). La investigación científica y la formación docente. Buenos Aires: Miño y Dávila.

- Souto, M. (2009). Complejidad y Formación Docente. En: Yuni, J. La formación docente. Complejidad y ausencias. Córdoba: Encuentro Grupo Ed.

- Souto, M. (2016). Pliegues de la Formación. Sentidos y herramientas para la formación docente. Rosario: Homo Sapiens.

- Terigi, F. (1996). Aportes para el debate curricular. Notas para otro análisis en la política curricular en la Argentina. Revista Novedades Educativas, Año 8. N66. pp. 1-9.

\section{Documentos de gobierno}

- Gobierno de Argentina. Ministerio de Cultura y Educación (1988). "Creación del Magisterio de Educación Básica". Resolución Ministerial №1679. Buenos Aires.

- Gobierno de Argentina, Ministerio de Cultura y Educación (1991). "Programa de transformación de la Formación Docente". № 2547/91.

- Gobierno de Argentina (1991). "Ley de Transferencias de Servicios Educativos 24.049/92", 2 de enero de 1992, Ministerio de Cultura y Educación, Buenos Aires.

- Gobierno de Argentina. "Programa Nacional de Formación Docente (2001). Informe de Gestión 2000-2001. Ministerio de Educación de la Nación Argentina.

- Anexo Programa Nacional de Formación Docente (2001). Informe de Gestión 20002001. Ministerio de Educación de la Nación Argentina. 